\title{
Optimization of the Luminosity Spectrum in the NLC
}

\author{
K. A. Thompson et al.
}

Presented at 1999 Particle Accelerator Conference, 3/29/99—4/2/99, New York, NY, USA 
SLAC-PUB-8088

May 1999

\title{
OPTIMIZATION OF THE LUMINOSITY SPECTRUM IN THE NLC
}

\author{
K. A. Thompson et al. \\ Stanford Linear Accelerator Center, Stanford University, Stanford, CA 94309
}

\begin{abstract}
The energy spectrum of electrons at the interaction point of a linear collider is deterimined largely by the beamstrahlung spectrum. The beamstrahlung spectrum in turn is sensitive to the design parameters at the interaction point. In this paper we examine the optimization of the luminosity spectrum for discovery and detailed exploration of various physics processes of interest in the NLC, in particular, top and stop pair production, and a class of processes occuring via $\mathrm{W}-\mathrm{W}$ scattering.
\end{abstract}

Stanford Linear Accelerator Center, Stanford University, Stanford, CA 94309

Work supported by Department of Energy contract DE-AC03-76SF00515. 


\title{
OPTIMIZATION OF THE LUMINOSITY SPECTRUM IN THE NLC*
}

\author{
$\underline{\text { K.A. Thompson }}^{\dagger}$, T.L. Barklow, T.W. Markiewicz, T.O.Raubenheimer, \\ Stanford Linear Accelerator Center, Stanford, CA 94309
}

\section{Abstract}

The energy spectrum of electrons at the interaction point of a linear collider is determined largely by the beamstrahlung spectrum. The beamstrahlung spectrum in turn is sensitive to the design parameters at the interaction point. In this paper we examine the optimization of the luminosity spectrum for discovery and detailed exploration of various physics processes of interest in the NLC, in particular, top and stop pair production, and a class of processes occuring via $\mathrm{W}-\mathrm{W}$ scattering.

\section{INTRODUCTION}

The energy spectrum of electrons at the interaction point of a linear collider is determined largely by the beamstrahlung spectrum (emission of photons due to the interaction of an electron with the collective field of the oncoming beam), with additional effects due to initial state radiation and energy spread in the beam coming from upstream. The beamstrahlung spectrum depends on the design parameters at the interaction point. Typically there is roughly half the luminosity very near the nominal center of mass energy, with the remaining luminosity distributed in a tail extending to significantly lower energies. While we realize various processes must be studied individually in greater detail than is presented here, our goal is to examine optimization tradeoffs for some important classes of physics processes in future linear colliders. In this paper, we focus mostly on processes with cross sections increasing with energy over the range of interest.

\section{NLC BASELINE DESIGNS}

We give some luminosity-related parameters for the basic NLC designs near (but not necessarily exactly at!) 500, 1000, $1500 \mathrm{GeV}$ c.m. energy in Tables 1, 2, and 3[1]. The values shown for the pinch enhancement $H_{D}$, number of beamstrahlung photons per incoming particle $n_{\gamma}$, and average beamstrahlung energy loss $\delta_{B}$ are obtained from simulations using the beam-beam code Guineapig[2] and agree to within a few percent with those obtained from analytic formulas in the literature[3].

Note that for a given design, the c.m. energy decreases with higher bunch charge $N$ because of beam loading, which due to costs is not completely compensated. There is also variation in other parameters among different design versions near a given energy. For purposes of understanding the physics tradeoffs, it is also useful to vary only one

\footnotetext{
* Work supported by Department of Energy Contract DE-AC0376SF00515.

† Email: kthom@SLAC.Stanford.edu
}

Table 1: IP parameters for three $\sim 1 / 2 \mathrm{TeV}$ c.m. NLC designs

\begin{tabular}{lrrr} 
& $\mathrm{A}-500$ & $\mathrm{~B}-500$ & $\mathrm{C}-500$ \\
\hline$E_{\text {beam }}[\mathrm{GeV}]$ & 267.5 & 257.5 & 250. \\
$\mathrm{~N}\left[10^{10}\right]$ & 0.75 & 0.95 & 1.1 \\
$\gamma \epsilon_{x} / \gamma \epsilon_{y}[\mu \mathrm{m}-\mathrm{r}]$ & $4.0 / 0.06$ & $4.5 / 0.1$ & $5.0 / 0.14$ \\
$\beta_{x} / \beta_{y}[\mathrm{~mm}]$ & $10 / 0.1$ & $12 / 0.12$ & $13 / 0.2$ \\
$\sigma_{z}[\mu \mathrm{m}]$ & 90. & 120. & 145. \\
$\sigma_{x} / \sigma_{y}[\mathrm{~nm}]$ & $276 / 3.4$ & $327 / 4.9$ & $365 / 7.6$ \\
$\Upsilon_{a v g}$ & 0.14 & 0.11 & 0.09 \\
$H_{D}$ & 1.36 & 1.30 & 1.49 \\
$n_{\gamma}$ & 1.08 & 1.18 & 1.24 \\
$\delta_{B}$ & $4.3 \%$ & $3.9 \%$ & $3.7 \%$ \\
Bunches/train & 95 & 95 & 95 \\
Rep. rate & 120 & 120 & 120 \\
$L_{D}\left[10^{33} \mathrm{~cm}^{-2} \mathrm{sec}^{-1}\right]$ & 7.42 & 6.66 & 5.94
\end{tabular}

Table 2: IP parameters for three $\sim 1 \mathrm{TeV}$ c.m. NLC designs

\begin{tabular}{lrrr} 
& $\mathrm{A}-1000$ & $\mathrm{~B}-1000$ & $\mathrm{C}-1000$ \\
\hline$E_{\text {beam }}[\mathrm{GeV}]$ & 523. & 504. & 489. \\
$\mathrm{~N}\left[10^{10}\right]$ & 0.75 & 0.95 & 1.1 \\
$\gamma \epsilon_{x} / \gamma \epsilon_{y}[\mu \mathrm{m}-\mathrm{r}]$ & $4.0 / 0.06$ & $4.5 / 0.1$ & $5.0 / 0.14$ \\
$\beta_{x} / \beta_{y}[\mathrm{~mm}]$ & $10 / 0.125$ & $12 / 0.15$ & $13 / 0.2$ \\
$\sigma_{z}[\mu \mathrm{m}]$ & 90. & 120. & 145. \\
$\sigma_{x} / \sigma_{y}[\mathrm{~nm}]$ & $198 / 2.7$ & $234 / 3.9$ & $261 / 5.4$ \\
$\Upsilon_{\text {avg }}$ & 0.39 & 0.30 & 0.25 \\
$H_{D}$ & 1.50 & 1.44 & 1.50 \\
$n_{\gamma}(\mathrm{sim})$ & 1.39 & 1.53 & 1.62 \\
$\delta_{B}(\mathrm{sim})$ & $9.5 \%$ & $9.2 \%$ & $8.7 \%$ \\
Bunches/train & 95 & 95 & 95 \\
Rep. rate & 120 & 120 & 120 \\
$L_{D}\left[10^{33} \mathrm{~cm}^{-2} \mathrm{sec}^{-1}\right]$ & 14.33 & 12.95 & 11.67
\end{tabular}

Table 3: IP parameters for two $1.5 \mathrm{TeV}$ c.m. NLC designs

\begin{tabular}{lrr} 
& $\mathrm{A}-1500$ & $\mathrm{~B}-1500$ \\
\hline$E_{\text {beam }}[\mathrm{GeV}]$ & 703 & 739 \\
$\mathrm{~N}\left[10^{10}\right]$ & 1.4 & 0.95 \\
$\gamma \epsilon_{x} / \gamma \epsilon_{y}[\mu \mathrm{m}-\mathrm{r}]$ & $4.5 / 0.14$ & $4.5 / 0.1$ \\
$\beta_{x} / \beta_{y}[\mathrm{~mm}]$ & $15 / 0.2$ & $13 / 0.2$ \\
$\sigma_{z}[\mu \mathrm{m}]$ & 130. & 150. \\
$\sigma_{x} / \sigma_{y}[\mathrm{~nm}]$ & $222 / 4.5$ & $201 / 3.7$ \\
$\Upsilon_{a v g}$ & 0.60 & 0.41 \\
$H_{D}$ & 1.61 & 1.50 \\
$n_{\gamma}$ & 2.2 & 1.7 \\
$\delta_{B}$ & $17 \%$ & $12 \%$ \\
Bunches/train & 95 & 95 \\
Rep. rate & 60 & 90 \\
$L_{D}\left[10^{33} \mathrm{~cm}^{-2} \mathrm{sec}^{-1}\right]$ & 14.3 & 12.3
\end{tabular}


Table 4: Fractional luminosities for NLC designs $500 \quad 1000 \quad 1500-\mathrm{A} \quad 1500-\mathrm{B}$

\begin{tabular}{lrrr}
\hline \hline beamst. only: & & & \\
$L_{99.5 \%}(\operatorname{sim})$ & $61 \%$ & $46 \%$ & $31 \%$ \\
$L_{99 \%}(\operatorname{sim})$ & $67 \%$ & $52 \%$ & $35 \%$ \\
$L_{98 \%}(\operatorname{sim})$ & $75 \%$ & $59 \%$ & $41 \%$ \\
$L_{95 \%}(\operatorname{sim})$ & $86 \%$ & $72 \%$ & $54 \%$ \\
$L_{90 \%}(\operatorname{sim})$ & $94 \%$ & $83 \%$ & $66 \%$ \\
$L_{80 \%}(\operatorname{sim})$ & $99 \%$ & $94 \%$ & $82 \%$ \\
$L_{50 \%}(\operatorname{sim})$ & $\sim 100 \%$ & $\sim 100 \%$ & $99 \%$ \\
& & & \\
beamst.+ISR: & & & \\
$L_{99.5 \%}(\operatorname{sim})$ & $37 \%$ & $27 \%$ & $18 \%$ \\
$L_{99 \%}(\operatorname{sim})$ & $44 \%$ & $33 \%$ & $22 \%$ \\
$L_{98 \%}(\operatorname{sim})$ & $54 \%$ & $41 \%$ & $28 \%$ \\
$L_{95 \%}(\operatorname{sim})$ & $69 \%$ & $56 \%$ & $41 \%$ \\
$L_{90 \%}(\operatorname{sim})$ & $81 \%$ & $70 \%$ & $55 \%$ \\
$L_{80 \%}(\operatorname{sim})$ & $91 \%$ & $84 \%$ & $73 \%$ \\
$L_{50 \%}(\operatorname{sim})$ & $98 \%$ & $97 \%$ & $95 \%$
\end{tabular}

parameter at a time (for instance, $N, \sigma_{z}, \beta_{x}, \beta_{y}, \gamma \epsilon_{y}$, or $\left.\gamma \epsilon_{x}\right)$. In doing such parameter variations, one finds[4] that the best "knobs" for increasing luminosity at the high energy end of the spectrum are $\gamma \epsilon_{y}$ and $N$. It is difficult to decrease $\gamma \epsilon_{y}$ very much, but one may consider increasing $N$ if one is willing either to let the energy drop or compensate for the beam-loading.

We show the luminosity spectra for the nominal designs, with beamstrahlung only and with both beamstrahlung and initial state radiation (ISR) in Table 4. The fractional luminosities are shown, e.g., $L_{99 \%}$ denotes the percentage of the luminosity with c.m. energy greater than or equal to 99\% of the nominal c.m. energy. (These numbers are not significantly different for the $\mathrm{A}, \mathrm{B}$, and $\mathrm{C}$ variations of the designs at 500 and $1000 \mathrm{GeV}$.)

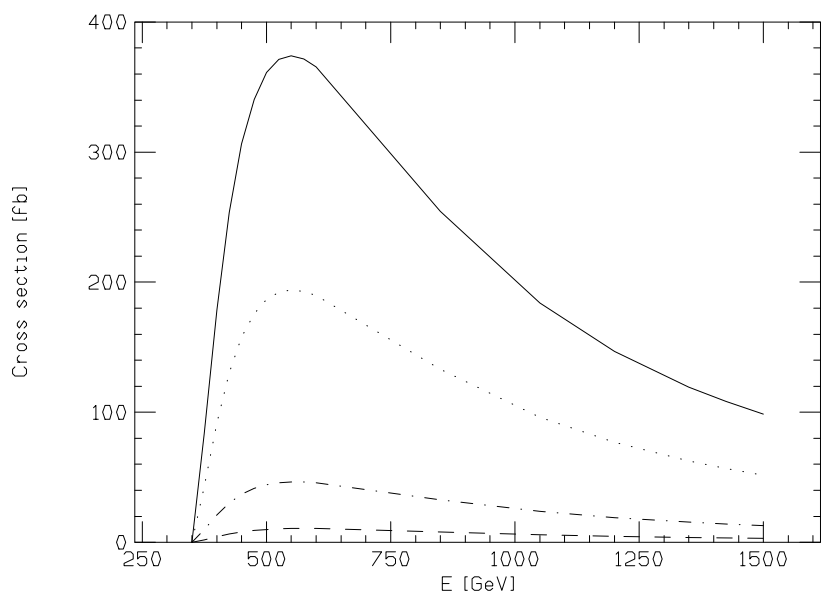

Figure 1: Cross sections for $e_{L}^{-} e_{R}^{+} \rightarrow \tilde{t}_{R} \tilde{\bar{t}}_{R}$ (dot-dashed curve). $e_{R}^{-} e_{L}^{+} \rightarrow \tilde{t}_{R} \tilde{\bar{t}_{R}}$ (dotted curve). $e_{L}^{-} e_{R}^{+} \rightarrow \tilde{t}_{L} \tilde{\bar{t}}_{L}$ (solid curve), $e_{R}^{-} e_{L}^{+} \rightarrow \tilde{t}_{L} \tilde{\bar{t}_{L}}$ (dashed curve).
$23 \%$

$36 \%$

$50 \%$

$64 \%$

$80 \%$

$97 \%$
Table 5: Number of top and stop pair production events in a $10^{7}$ second running year, for nominal designs near $1 / 2 \mathrm{TeV}$

\begin{tabular}{lrrr} 
c.m. & $\mathrm{A}-500$ & $\mathrm{~B}-500$ & $\mathrm{C}-500$ \\
\hline$e_{L}^{-} e_{R}^{+} \rightarrow t \bar{t}$ & 785000 & 674000 & 581000 \\
$e_{R}^{-} e_{L}^{+} \rightarrow t \bar{t}$ & 357000 & 305000 & 262000 \\
$e_{L}^{-} e_{R}^{+} \rightarrow \tilde{t}_{R} \tilde{t_{R}}$ & 22000 & 17500 & 14200 \\
$e_{R}^{-} e_{L}^{+} \rightarrow \tilde{t}_{R} \tilde{t_{R}}$ & 92000 & 74000 & 60200 \\
$e_{L}^{-} e_{R}^{+} \rightarrow \tilde{t}_{L} \tilde{t}_{L}$ & 178000 & 143000 & 116500 \\
$e_{R}^{-} e_{L}^{+} \rightarrow \tilde{t}_{L} \tilde{\bar{t}_{L}}$ & 4900 & 3900 & 3200
\end{tabular}

Table 6: Number of top and stop pair production events in a $10^{7}$ second running year, for modified B-500 design (varying $N$ and keeping energy fixed).

\begin{tabular}{lrrr}
$N\left[10^{10}\right]$ & 1.1 & 1.3 & 1.5 \\
\hline$e_{L}^{-} e_{R}^{+} \rightarrow t \bar{t}$ & 927000 & 1347000 & 1856000 \\
$e_{R}^{-} e_{L}^{+} \rightarrow t \bar{t}$ & 420000 & 609000 & 837700 \\
$e_{L}^{-} e_{R}^{+} \rightarrow \tilde{t}_{R} \tilde{t_{R}}$ & 23900 & 34000 & 46000 \\
$e_{R}^{-} e_{L}^{+} \rightarrow \tilde{t}_{R} \tilde{t_{R}}$ & 101000 & 144000 & 195000 \\
$e_{L}^{-} e_{R}^{+} \rightarrow \tilde{t}_{L} \tilde{t}_{L}$ & 195000 & 279000 & 377000 \\
$e_{R}^{-} e_{L}^{+} \rightarrow \tilde{t}_{L} \tilde{\bar{t}_{L}}$ & 5300 & 7600 & 10200
\end{tabular}

\section{SUSY SCALAR PRODUCTION}

We compare the rates of top and stop production in a 500$\mathrm{GeV}$ collider, assuming $m_{\tilde{t}}=m_{t}$. The total top and stop pair production cross-sections are plotted in Figures 2 and 1 respectively.

The production rates (\# events in a running year of $10^{7}$ sec) are shown in Table 5 for the three nominal designs near $1 / 2 \mathrm{TeV}$ c.m. energy. The rates with bunch charge $N$ pushed up from the nominal B-500 design value are shown in Table 6. Here we assume it is reasonable to compensate the extra beam loading to keep the energy fixed.

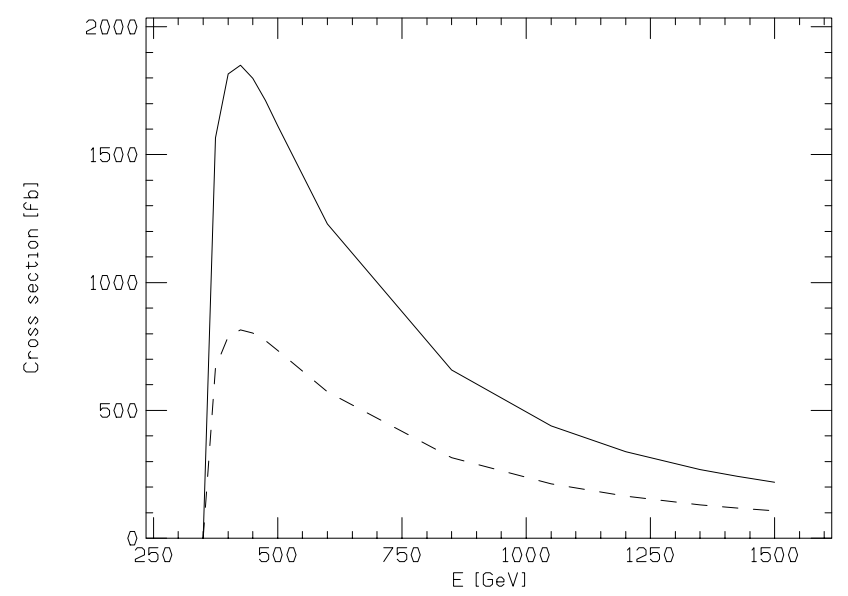

Figure 2: Cross sections for $e_{L}^{-} e_{R}^{+} \rightarrow t \bar{t}$ (solid curve) and $e_{R}^{-} e_{L}^{+} \rightarrow t \bar{t}$ (dashed curve). 
Table 7: $e^{+} e^{-} \rightarrow \nu \bar{\nu} t \bar{t} ; \quad M_{H}=100 \mathrm{GeV}$; No polarization No. of \% change $n_{\gamma}$ events B-1000

\begin{tabular}{lccc}
\hline $\mathrm{A}-1000$ & 2800 & $+26 \%$ & 1.4 \\
$\mathrm{~B}-1000$ & 2220 & $0 \%$ & 1.5 \\
$\mathrm{C}-1000$ & 1780 & $-20 \%$ & 1.6 \\
$\mathrm{~B}-1000, \gamma \epsilon_{y} \downarrow 0.08 \mu \mathrm{m}-\mathrm{r}$ & 2520 & $+13 \%$ & 1.5 \\
$\mathrm{~B}-1000, \gamma \epsilon_{y} \downarrow 0.06 \mu \mathrm{m}-\mathrm{r}$ & 2960 & $+33 \%$ & 1.5 \\
$\mathrm{~B}-1000, \gamma \epsilon_{y} \downarrow 0.04 \mu \mathrm{m}-\mathrm{r}$ & 3780 & $+70 \%$ & 1.5 \\
$\mathrm{~B}-1000, \mathrm{~N} \uparrow 1.1 \cdot 10^{10}$ & 2990 & $+35 \%$ & 1.8 \\
$\mathrm{~B}-1000, \mathrm{~N} \uparrow 1.3 \cdot 10^{10}$ & 4200 & $+89 \%$ & 2.0 \\
$\mathrm{~B}-1000, \mathrm{~N} \uparrow 1.5 \cdot 10^{10}$ & 5620 & $+153 \%$ & 2.3 \\
$\mathrm{~B}-1000, \mathrm{~N} \uparrow 1.1 \cdot 10^{10}, \mathrm{E} \downarrow 978$ & 2670 & $+20 \%$ & 1.8 \\
$\mathrm{~B}-1000, \mathrm{~N} \uparrow 1.3 \cdot 10^{10}, \mathrm{E} \downarrow 935$ & 3110 & $+40 \%$ & 2.0 \\
$\mathrm{~B}-1000, \mathrm{~N} \uparrow 1.5 \cdot 10^{10}, \mathrm{E} \downarrow 891$ & 3410 & $+54 \%$ & 2.3 \\
$\mathrm{~B}-1000, \beta_{y} \uparrow 0.30 \mathrm{~mm}$ & 1910 & $-14 \%$ & 1.5
\end{tabular}

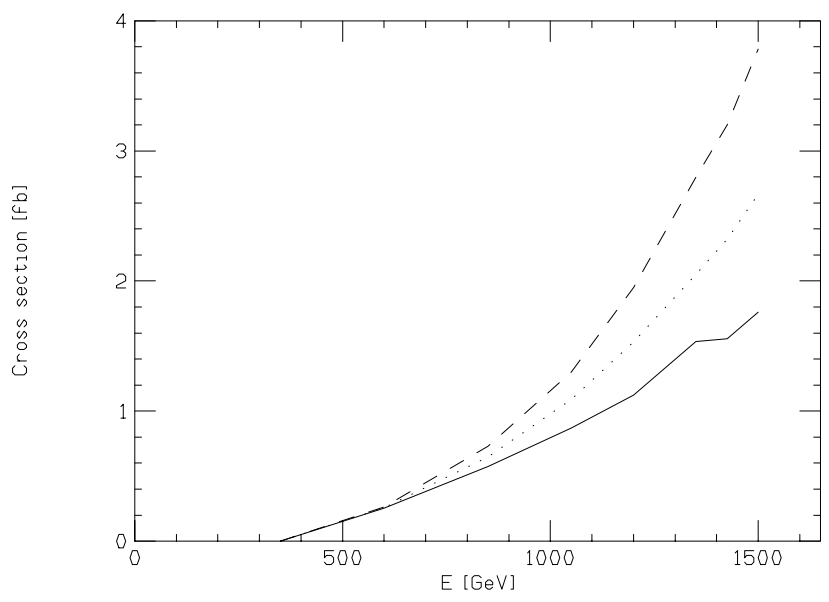

Figure 3: Cross sections for $e^{+} e^{-} \rightarrow \nu_{e} \bar{\nu}_{e} t \bar{t}$ for $M_{H}=$ $100 \mathrm{GeV}$ (solid curve), $M_{H}=1000 \mathrm{GeV}$ (dashed curve), $M_{H}=\infty$ (dotted curve).

\section{WW-SCATTERING PROCESSES}

The class of processes $e^{+} e^{-} \rightarrow \ell \ell X$ occurring via $W W$ scattering, where one or both $\ell$ 's is a neutrino and $X$ could be for example $t \bar{t}, W$ 's and/or $Z$ 's, have cross sections which are rising with energy through the $\mathrm{TeV}$ energy scale. Taking as a representative example the process $e^{+} e-\rightarrow \nu_{e} \bar{\nu}_{e} t \bar{t}$, (see Figure 3) we show the number of events in $10^{7}$ seconds of running for designs near $1 \mathrm{TeV}$ in Table 7. In addition to the nominal A, B, and $\mathrm{C}$ designs, we show the increase in luminosity attainable with $\gamma \epsilon_{y}$ decreased from its nominal value of $0.1 \mu \mathrm{m}-\mathrm{r}$, or with $N$ increased from its nominal value of $0.95 \times 10^{10}$, with and without compensation of the extra beam-loading. The biggest gain is to be had by increasing $N$ and keeping the energy fixed; note also that further study is needed to see how high $n_{\gamma}$ can be allowd to go. We have also noted[4] that substantially increasing $\beta_{y}$ (which relaxes machine tolerances) does not reduce luminosity very much; for our example we see only a $14 \%$ reduction in the number of events
Table 8: $e^{+} e^{-} \rightarrow \nu \bar{\nu} t \bar{t} ; \quad M_{H}=100 \mathrm{GeV}$; No polarization

\begin{tabular}{lccc} 
& $\begin{array}{c}\text { No. of } \\
\text { events }\end{array}$ & $\begin{array}{c}\% \text { change } \\
\text { B-1500 }\end{array}$ & $n_{\gamma}$ \\
\hline $\mathrm{A}-1500$ & 8610 & $-9 \%$ & 2.2 \\
$\mathrm{~B}-1500$ & 9420 & $0 \%$ & 1.7 \\
$\mathrm{~B}-1500, \mathrm{~N} \uparrow 1.1 \cdot 10^{10}$ & 12800 & $+36 \%$ & 2.0 \\
$\mathrm{~B}-1500, \mathrm{~N} \uparrow 1.3 \cdot 10^{10}$ & 18000 & $+90 \%$ & 2.3 \\
$\mathrm{~B}-1500, \mathrm{~N} \uparrow 1.5 \cdot 10^{10}$ & 23900 & $+154 \%$ & 2.6 \\
$\mathrm{~B}-1500, \beta_{y} \uparrow 0.30 \mathrm{~mm}$ & 8700 & $-8 \%$ & 1.7
\end{tabular}

when $\beta_{y}$ is doubled from its nominal value. Similar results for designs near $1.5 \mathrm{TeV}$ are shown in Table 8 .

\section{DISCUSSION AND CONCLUSIONS}

We have not discussed the very important issue of detector backgrounds in this paper - our general philosophy is that when total rate for the various processes considered here is optimized, one will indeed end up with a larger number of useful events even after appropriate cuts are applied to reduce backgrounds. However, we mention some issues that must be kept in mind when designing and optimizing the interaction region and detector. For example, the process $\gamma \gamma \rightarrow W W$ (where the $\gamma$ 's are bremsstrahlung photons) is a significant background to $e^{+} e-\rightarrow \nu \bar{\nu} W W$, since the $e^{ \pm}$ that radiate the photons are typically close to the beam axis and may escape tagging. For these electroweak symmetry breaking studies, electron tagging capability down to about $150 \mathrm{mrad}$ is needed[5]. Other processes, for instance some SUSY parameter determinations [6] as well as studies of the perturbative QCD pomeron (BFKL dynamics) in $\gamma^{*} \gamma^{*} \rightarrow$ hadrons $[7,8]$ have even more stringent angular coverage requirements, down to about $40 \mathrm{mrad}$.

We thank Lance Dixon and Michael Peskin for useful discussions and comments.

\section{REFERENCES}

[1] See http://www-project.slac.stanford.edu/lc/nlc-tech.html under Accelerator Physics parameters.

[2] D.Schulte, Ph.D. thesis, 1996.

[3] Yokoya,K. and Chen,P., in M.Dienes,et.al. (ed.), Frontiers of Particle Beams: Intensity Limitations (Springer-Verlag, 1988).

[4] K.Thompson and T.Raubenheimer, NLC collider note LCC0014 (1999).

[5] V.Barger, K.Cheung, T.Han, R.Phillips, Phys.Rev.D., 52, 3815 (1995).

[6] N.Danielson and E.Goodman, COLO-HEP-422, unpublished.

[7] S.Brodsky, F.Hautmann, and D.Soper, 5th International Workshop on Deep Inelastic Scattering and QCD, Chicago, 14-18 April, 1997, hep-ph/9707444.

[8] J.Bartels, A.DeRoeck, and H.Lotter, hep/9710500. 